\title{
The use of computerized physician order entry with clinical decision support reduces practice variance in ordering preoperative investigations: a retrospective cohort study
}

$\underline{\text { Sim Yilin Eileen }}{ }^{1}, \underline{\text { Tan Daryl Jian An }}{ }^{2}$, Abdullah Hairil Rizal ${ }^{1}$

1. Department of Anaesthesia, Singapore General Hospital; 2. Yong Loo Lin School of Medicine, National University of Singapore

\section{Background/Introduction}

Over-ordering of routine preoperative investigations is prevalent despite the presence of institutional guidelines to reduce variances in physician ordering practices. Our institution integrated a Clinical Decision Support (CDS) model into our hospital's Computerized Physician Ordering Entry (CPOE) system to guide physician orders. We aim to investigate if the implementation of CDS into CPOE increases physician adherence to our institutional guidelines.

\section{Methodology}

- Retrospective cohort study over 18 months, involving 11,792 patients

- Examine ordering patterns of physicians for preop investigations: FBC, renal panel, coagulation panel, CXR, ECG for elective surgeries.

- Orders = 'match' if they followed guidelines; 'over' if they were not required by guidelines, and 'under' if they were required but not ordered.

- Pre-implementation - first 10 months; 7,911 patients

- Electronic Admission Form with Clinical Decision Support (CDS) was implemented on the $11^{\text {th }}$ month, in phases over 1 month

- Post-implementation - assessed from $13^{\text {th }}$ to $18^{\text {th }}$ month, 3,815 patients

\section{Results}

- Both pre-implementation group and postimplementation group were of similar demographic profile and surgical casemix ( $p>0.05)$.

- In both groups, healthier, ASA 1 patients had the lowest frequency of matched orders. The matched frequency increases with higher ASA score.

- Within each ASA group, there was at least $1.5 \mathrm{x}$ more over-orders to under-orders.

- In the pre-implementation group

- FBC showed the highest match rate (96.3\%), followed by RP (90.5\%), CP (65.2), CXR (60.8\%) and ECG (56.4\%).

- Highest incidence of over-ordering in the preimplementation group was found in CXR (35.7\%) and ECG (30.4\%)

- Highest incidence of under-ordering in the preimplementation group was found in CP (22.9\%).

\section{Effect of EAF with CDS implementation}

\section{Amongst ASA classification (Table 1)}

Significant improvement in matched orders after implementation of CDS

- ASA 1 patients $(62.2 \%$ to $67.1 \%(+4.9 \%, \mathrm{P}<0.01)$

- ASA 2 patients $(74.7 \%$ to $79.1 \%,+4.4 \%, p<0.001)$

- ASA 3 patients $(82.2 \%$ to $84.5 \%,+2.3 \%, p=0.006)$.

- ASA 4 patients, matched orders decreased from $88.2 \%$ to $82.0 \%,-6.2 \%, P=0.252$. However this was not significant due to the small number of patients $(\mathrm{N}=220)$.
Table 1. Change in Match, Under-order and Over-order rates according to ASA score $50 \quad 100$

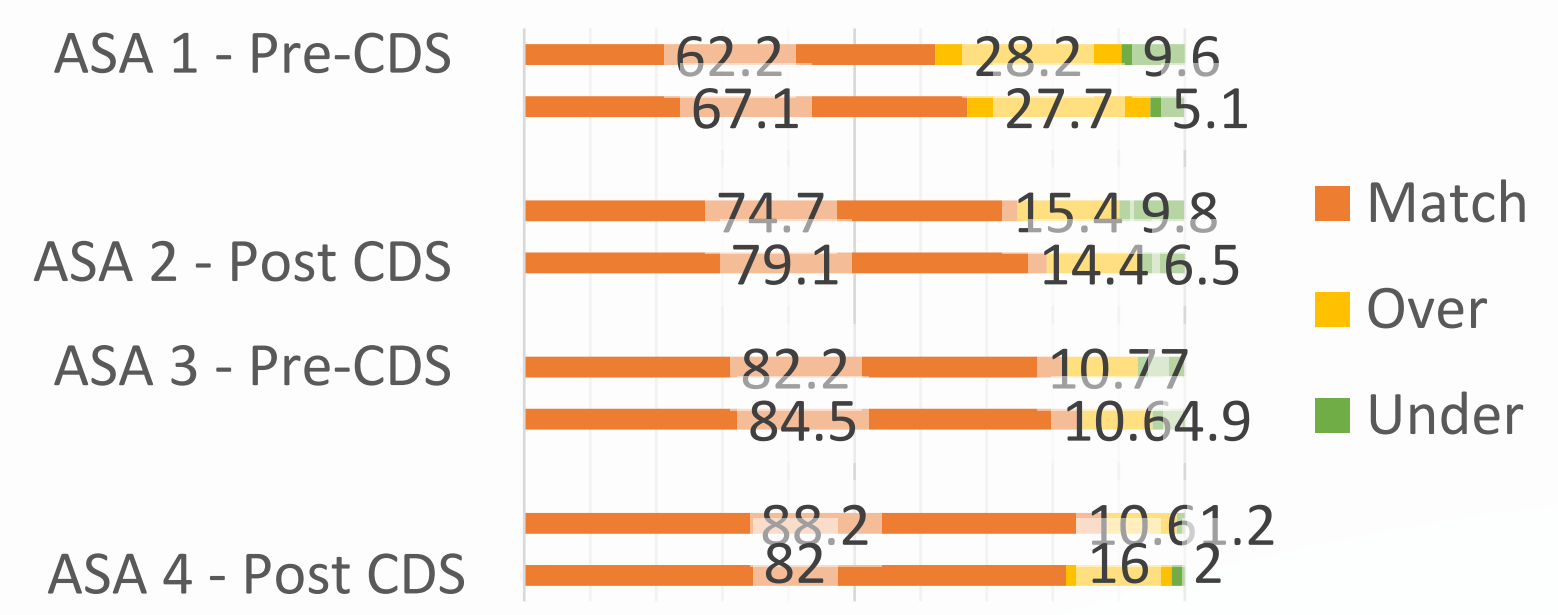

\section{Within Orders}

Improvement in Match rate

- Greatest improvement in CP $(+12.0 \%)$

- $\operatorname{RP}(+1.0 \%, \mathrm{P}<0.001)$, ECG $(+3.1 \%, \mathrm{P}=0.007)$ and $\mathrm{CXR}$ $(+3.8 \%, \quad P<0.001)$ also showed small but significant improvement.

- $\mathrm{FBC}$ had an insignificant decrease in match rate $(-0.3 \%$, $\mathrm{P}=0.106)$

Reduction in Under Orders

- Present in all, but greatest reduction in under orders was found in CP $(-11.1 \%, P<0.001)$

Table 2. Change in Match, Under-order and Over-order rates according to Individual Investigations

$$
\begin{array}{llllll}
0 & 20 & 40 & 60 & 80 & 100
\end{array}
$$

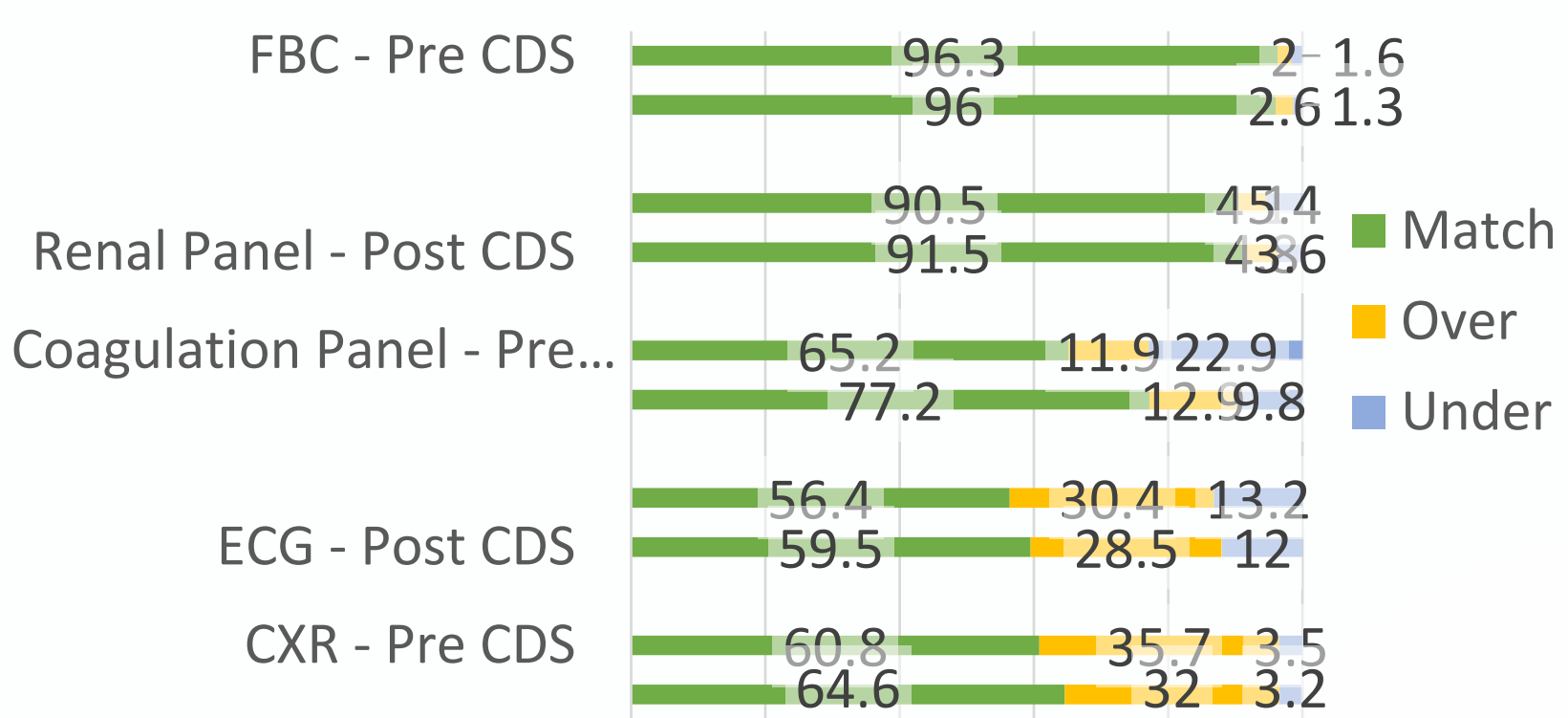

